\title{
BMJ Open Screening for cancer in patients with unprovoked venous thromboembolism: protocol for a systematic review and individual patient data meta-analysis
}

\author{
Nick van Es, ${ }^{1}$ Grégoire Le Gal, ${ }^{2,3}$ Hans-Martin Otten, ${ }^{4}$ Philippe Robin, ${ }^{5}$ \\ Andrea Piccioli, ${ }^{6}$ Ramon Lécumberri, ${ }^{7}$ Luis Jara Palomares, ${ }^{8}$ Piotr Religa,,${ }^{9,10}$ \\ Viriginie Rieu, ${ }^{11}$ Matthew T. Rondina, ${ }^{12}$ Mariëlle M Beckers, ${ }^{13}$ Paolo Prandoni, ${ }^{6}$ \\ Pierre-Yves Salaun, ${ }^{5}$ Marcello Di Nisio, ${ }^{1,14}$ Patrick M Bossuyt, ${ }^{15}$ Harry R Büller, ${ }^{1}$ \\ Marc Carrier $^{2}$
}

To cite: van Es N, Gal GL, Otten H-M, et al. Screening for cancer in patients with unprovoked venous thromboembolism: protocol for a systematic review and individual patient data meta-analysis. BMJ Open 2017;7:e015562. doi:10.1136/ bmjopen-2016-015562

- Prepublication history and additional material are available. To view these files, please visit the journal online (http://dx.doi.org/ 10.1136/ bmjopen-2016-015562)

Received 15 December 2016 Revised 3 March 2017 Accepted 31 March 2017

CrossMark

For numbered affiliations see end of article.

Correspondence to Marc Carrier; mcarrier@toh.ca

\section{ABSTRACT}

Introduction 0ccult cancer is present in $4 \%-9 \%$ of patients with unprovoked venous thromboembolism (VTE). Screening for cancer may be considered in these patients, with the aim to diagnose cancers in an early, potentially curable stage. Information is needed about the risk of occult cancer, overall and in specific subgroups, additional risk factors and on the performance of different screening strategies.

Methods and analysis MEDLINE, Embase and CENTRAL databases were searched from November 2007 to January 2016 for prospective studies that had evaluated protocol-mandated screening for cancer in patients with unprovoked VTE and with at least 12 months' followup. Two reviewers independently assessed articles for eligibility. Ten eligible studies were identified and individual patient data were obtained from each of them. Study quality was assessed using the Quality Assessment of Diagnostic Accuracy Studies-2 tool . Generalised linear mixed-effects models was used to calculate estimates in a one-stage meta-analytic approach, overall and in a number of subgroups, including patients undergoing limited screening only, elderly patients, patients with previous VTE, smokers and patients using oestrogens. Ethics and dissemination Ethical approval is not required for this systematic review and individual patient data meta-analysis. Findings have been submitted for publication in peer-reviewed journals and presentations at national and international conferences to provide clinicians and other decision-makers with valid and precise risk estimates of occult cancer, overall and in specific clinical subgroups, with risk factors for occult cancer, with estimates of the diagnostic performance of limited screening and with an exploration of the benefit of extensive screening strategies.

\section{INTRODUCTION}

Unprovoked venous thromboembolism (VTE) may be the first sign of an occult cancer. Among patients with unprovoked VTE, approximately $4 \%-9 \%$ of patients are

\section{Strengths and limitations of this study}

- An unprovoked venous thromboembolism can be the first sign of an occult cancer.

- Various studies have reported different prevalences as well as variable yields for the different screening strategies.

- An individual patient data meta-analysis will allow to provide precise estimates for these important knowledge gaps.

diagnosed with cancer within the first year following VTE diagnosis. ${ }^{1-4}$ Given this high risk of cancer detection, clinicians have considered screening these patients for underlying occult cancers with the aim to detect the cancers in an early, potentially curable stage, which might reduce or even prevent cancer-related morbidity and mortality. However, the appropriate components and extent of the occult cancer screening strategy remain unclear.

Recently, various studies have independently shown that an extensive cancer screening including a CT of the abdomen/ pelvis or whole-body positron emission tomography (PET)/CT does not appear to have significant benefits over a more limited screening strategy. ${ }^{1-4}$ It is now suggested that a thorough medical history and physical examination, basic blood work, chest radiograph and age-specific and gender-specific testing according to national guidelines is an adequate occult cancer screening strategy for patients with unprovoked VTE.

Despite these new findings, several clinically relevant questions remain unanswered. The reported prevalence of occult cancer among patients with unprovoked VTE varied widely 


\begin{tabular}{|c|c|}
\hline 1 & $\begin{array}{l}\text { 'Thrombosis'(MeSH) OR 'pulmonary embolism'(MeSH) OR thrombos*(tiab) OR thrombot*(tiab) OR thromboemboli*(tiab) } \\
\text { OR phlebothrombosis(All Fields) OR 'deep vein thrombosis'(All Fields) OR pulmonary emboli*(All Fields) OR venous } \\
\text { thromboembolic event*(All Fields) }\end{array}$ \\
\hline 2 & $\begin{array}{l}\text { Neoplasms(MeSH) OR cancer(All Fields) OR neopl*(tiab) OR tumour (tiab) OR tumour(tiab) OR cancer (tiab) OR ‘Medical } \\
\text { Oncology'(MeSH) OR oncol`(tiab) OR ‘Hematologic Neoplasms'(MeSH) }\end{array}$ \\
\hline 3 & ‘Early Diagnosis'(MeSH) OR ‘Mass Screening’(MeSH) OR screen*(tiab) OR occult(tiab) \\
\hline 4 & '2007/11/01’(PDAT): ‘3000/12/31’(PDAT) \\
\hline 5 & 1 AND 2 AND 3 AND 4 \\
\hline
\end{tabular}

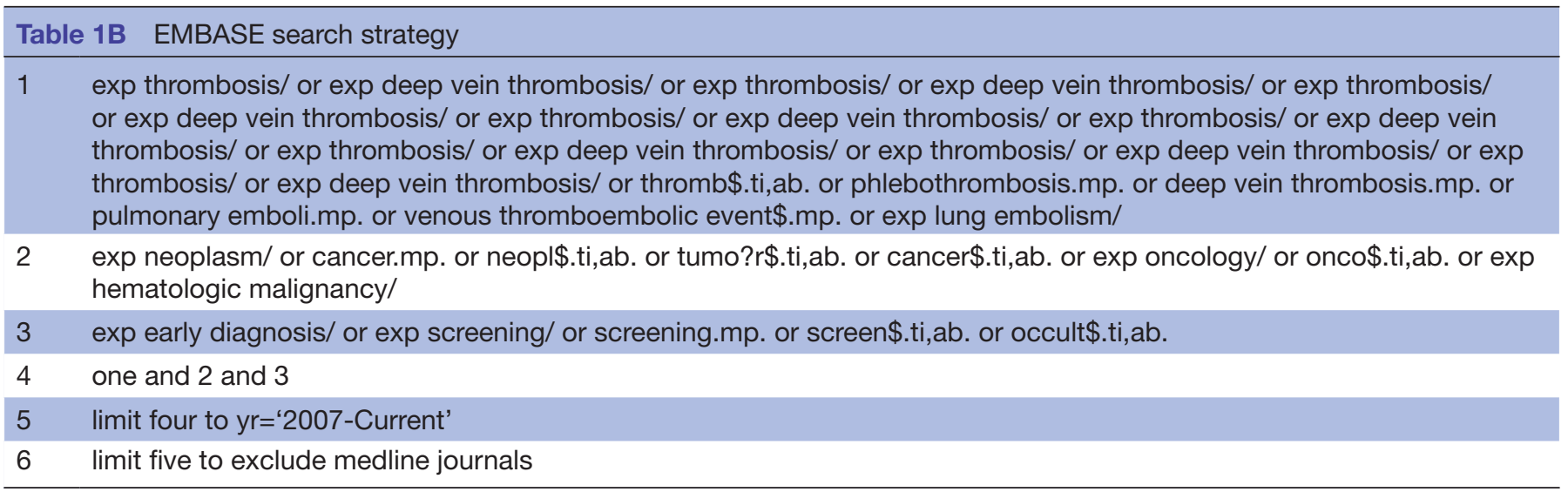

\section{Table 1C CENTRAL search strategy}

1 'MeSH descriptor: (Thrombosis) explode all trees' or 'MeSH descriptor: (Pulmonary Embolism) explode all trees' or thromb*:ti,ab or phlebothrombosis or 'deep vein thrombosis' or 'pulmonary embolism' or 'venous thromboembolic event*'

2 'MeSH descriptor: (Neoplasms) explode all trees' or 'MeSH descriptor: (Medical Oncology) explode all trees' or tumour*:ti,ab or tumour*:ti,ab or onco*:ti,ab or neopl*:ti,ab or cancer*:ti,ab

3 'MeSH descriptor: (Early Diagnosis) explode all trees' or 'MeSH descriptor: (Mass Screening) explode all trees' or screen $^{*}: t i, a b$ or occult*:ti,ab

$4 \quad\{$ AND \#1-\#3\} (limited to: Publication Year from 2008 in Trials)

across the different recently published studies. While the 1 year risk of cancer was $3.9 \%$ in the Screening for Occult Malignancy in Patients with Idiopathic Venous Thromboembolism (SOME) trial, ${ }^{3}$ Prandoni et al have reported a higher risk of $9.2 \% .{ }^{4}$ Clinicians need to have a more precise short-term and long-term risk estimate in order to properly counsel patients with unprovoked VTE and establish the need for additional screening investigations.

Given the fact that some recent clinical practice guidelines now suggest to use a more limited occult cancer screening strategy in this patient population, more detailed information about its positive predictive value and the proportion of false positive findings are needed to avoid possible complications from unnecessary additional investigations, anxiety for patients and increase in healthcare costs.

Establishing risk factors for occult cancer detection could lead to identification of high-risk patients in whom extra attention may be warranted. Furthermore, the impact of occult cancer screening on cancer-related mortality urgently needs further evaluation. While the early Subsequent diagnosis Of Malignancy in patients presentingwith Idiopathic venous Thromboembolism (SOMIT) trial suggested that a more extensive occult cancer screening strategy might decrease cancer-related mortality after 2 years of follow-up, other recently published studies could not confirm this finding. ${ }^{1-3}$ The short follow-up and lack of power of the individual studies may have precluded a proper assessment of the effect of occult cancer screening on cancer-related mortality in patients with unprovoked VTE. In addition, information is needed about the diagnostic components included in the different screening strategies, such as laboratory and imaging tests, in order to identify the tests with the highest yield for cancer detection.

To address these knowledge gaps, we sought to perform a systematic review and individual patient data meta-analysis (IPDMA) of contemporary studies evaluating cancer screening in patients with unprovoked VTE.

\section{METHODS AND ANALYSIS}

This protocol followed the recommendations in the statement on Preferred Reporting Items for Systematic 
Table 2 Reasons for exclusion

\begin{tabular}{|c|c|c|c|c|}
\hline First author & Title & Journal & Year & Reason for exclusion \\
\hline Bellas Beceiro & $\begin{array}{l}\text { Diagnosis of venous thromboembolism increases } \\
\text { probability of cancer detection for succeeding } \\
12 \text { months, but lacks the benefits and risk of } \\
\text { exhaustive screening (Spanish) }\end{array}$ & $\begin{array}{l}\text { FMC-Formación } \\
\text { Médica Continuada } \\
\text { en Atención Primaria }\end{array}$ & 2009 & $\begin{array}{l}\text { Not original data } \\
\text { (systematic review) }\end{array}$ \\
\hline de Oliveira & $\begin{array}{l}\text { Chest radiography as a method of screening for } \\
\text { occult cancer in patients with idiopathic deep vein } \\
\text { thrombosis }\end{array}$ & Angiology & 2012 & Retrospective design \\
\hline Galanaud & $\begin{array}{l}\text { Impact of anatomical location of lower limb } \\
\text { venous thrombus on the risk of subsequent cancer }\end{array}$ & $\begin{array}{l}\text { Thrombosis and } \\
\text { Haemostasis }\end{array}$ & 2014 & $\begin{array}{l}\text { No protocol-mandated } \\
\text { screening }\end{array}$ \\
\hline $\mathrm{Ge}$ & $\begin{array}{l}\text { Analysis of } 44 \text { cases of malignant tumours first } \\
\text { characterised by veinthrombosis (Chinese) }\end{array}$ & $\begin{array}{l}\text { Chinese Journal of } \\
\text { Cancer Prevention } \\
\text { and Treatment }\end{array}$ & 2010 & $\begin{array}{l}\text { Article not available } \\
\text { and author contact } \\
\text { information unknown }\end{array}$ \\
\hline Hildeyard & $\begin{array}{l}\text { Is there a benefit in CT screening for cancer in } \\
\text { patients with unprovoked proximal deep venous } \\
\text { thrombosis? A cohort study in the Oxford } \\
\text { University Hospitals NHS Trust. }\end{array}$ & $\begin{array}{l}\text { British Journal of } \\
\text { Haematology }\end{array}$ & 2016 & $\begin{array}{l}\text { No protocol-mandated } \\
\text { screening }\end{array}$ \\
\hline Ly & $\begin{array}{l}\text { Idiopathic venous thromboembolism: should we } \\
\text { screen extensively for cancer? (French) }\end{array}$ & $\begin{array}{l}\text { Sang Thrombose } \\
\text { Vaisseaux }\end{array}$ & 2012 & $\begin{array}{l}\text { Not original data } \\
\text { (narrative review) }\end{array}$ \\
\hline Mwirigi & $\begin{array}{l}\text { Is there benefit in extensive screening for } \\
\text { cancer in patients with unprovoked venous } \\
\text { thromboembolism? }\end{array}$ & Thrombosis Research & 2015 & Retrospective design \\
\hline Sorensen & $\begin{array}{l}\text { Venous thromboembolism and subsequent short- } \\
\text { term risk of an occult cancer }\end{array}$ & $\begin{array}{l}\text { Journal of } \\
\text { Thrombosis and } \\
\text { Haemostasis }\end{array}$ & 2008 & $\begin{array}{l}\text { Not original data } \\
\text { (editorial) }\end{array}$ \\
\hline
\end{tabular}

Review and Meta-Analyses Protocol (see online supplementary table 1). ${ }^{6}$ For the IPDMA, we adhered to the Preferred Reporting Items for Systematic Review and Meta-Analyses of Individual Participant Data. ${ }^{7}$

\section{Eligibility criteria}

We included randomised controlled trials, including controlled (non-randomised) trials, and prospective cohort studies. Studies that started enrolment of patients before 1 January 2000 were excluded from the systematic review. Important differences in clinical practice before 2000 including advances in cancer treatment, improved primary care regarding age- and gender-specific cancer screening and difference in performance of diagnostic tests (eg, CT), led to the decision to exclude earlier studies. Retrospective cohort studies and case-control studies were excluded.

Studies had to include consecutive adults (aged 18 years or older) with objectively confirmed, symptomatic lower extremity deep vein thrombosis (DVT) (proximal or distal) or pulmonary embolism (PE) in the absence of major provoking factors, such as recent surgery or immobilisation, known cancer and pregnancy. The study definitions of unprovoked VTE were accepted.

Patients had to receive protocol-mandated screening for occult cancer consisting of at least medical history, physical examination, basic blood work, a chest X-ray and/or age-specific and gender-specific testing (ie, limited screening). Patients had to be followed prospectively for cancer for a minimum of 12 months. There were no restrictions by type of setting or by language.

\section{Search strategy}

A previously reported systematic literature search $^{8}$ was updated by searching the Embase, MEDLINE and Cochrane Central Register of Controlled Trials databases from 1 November 2007 until 19 January 2016, combining terms for venous thromboembolism, cancer and screening. The MEDLINE search strategy was developed and discussed by four of the authors (NvE, H-MO, MDN, HRB and MC) and was then adapted to the syntax and subject headings of the other databases. The full search strategy is provided in tables $1 \mathrm{~A}, \mathrm{~B}$ and $\mathrm{C}$. In addition, conference proceedings of the International Society 


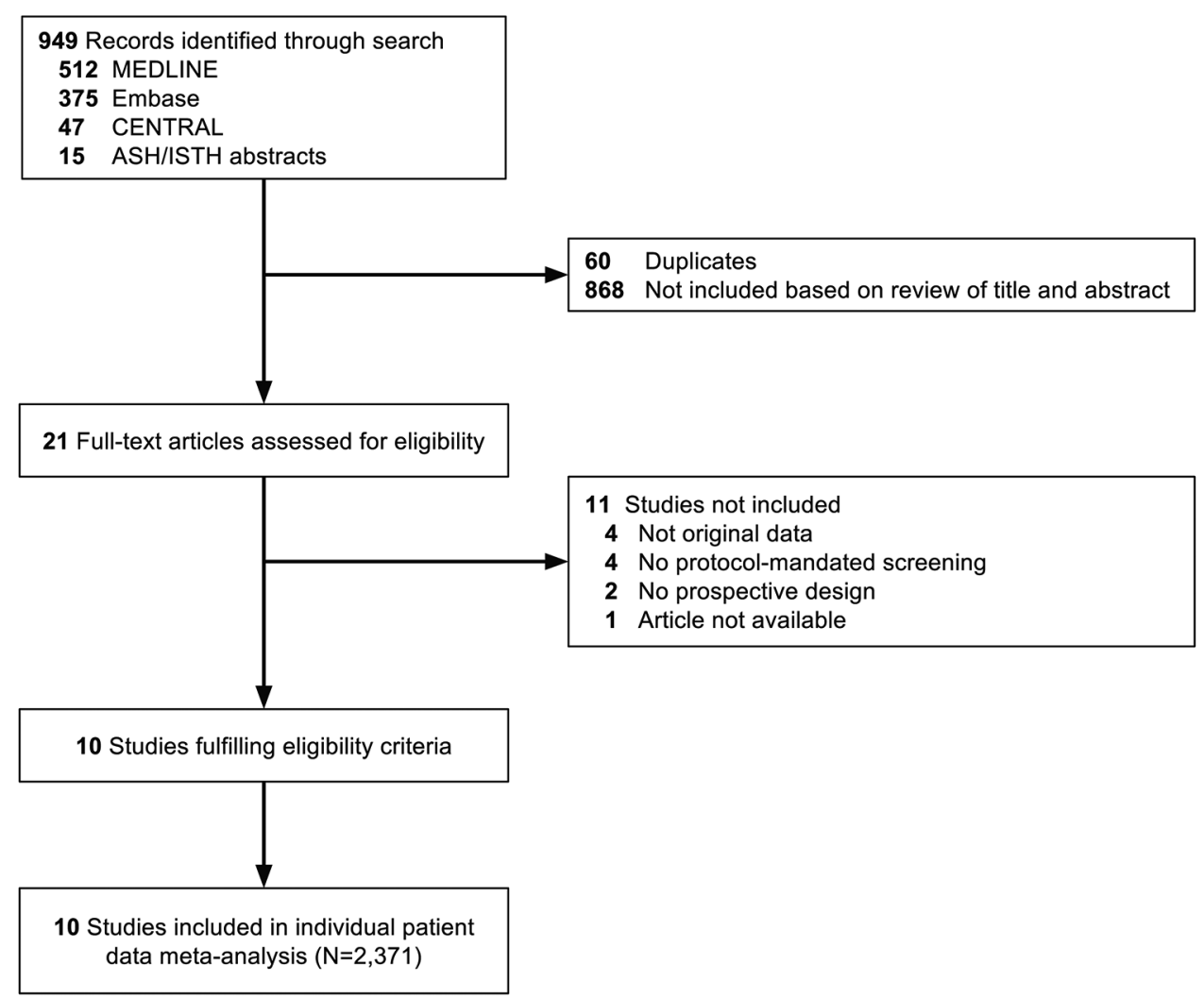

Figure 1 Preferred Reporting Items for Systematic Review and Meta-Analyses flow chart of systematic review.

on Thrombosis and Haemostasis and American Society of Hematology from 2007 to 2015 as well as references of eligible articles were hand-searched. There were no restrictions by language.

Literature search results were imported into EndNote V.17.3.1.8614, deduplicated and then uploaded to the Covidence platform (www.covidence.org) to facilitate collaboration among the reviewers during the study selection process. Two reviewers ( $\mathrm{NvE}$ and $\mathrm{MC}$ ) independently screened titles and abstracts and independently assessed the full-text articles for eligibility, using a predefined list of exclusion criteria. Disagreements were resolved by consensus. Neither of the review authors were blind to the journal titles or to the study authors or institutions.

Table 3 Studies included in the individual patient data meta-analysis

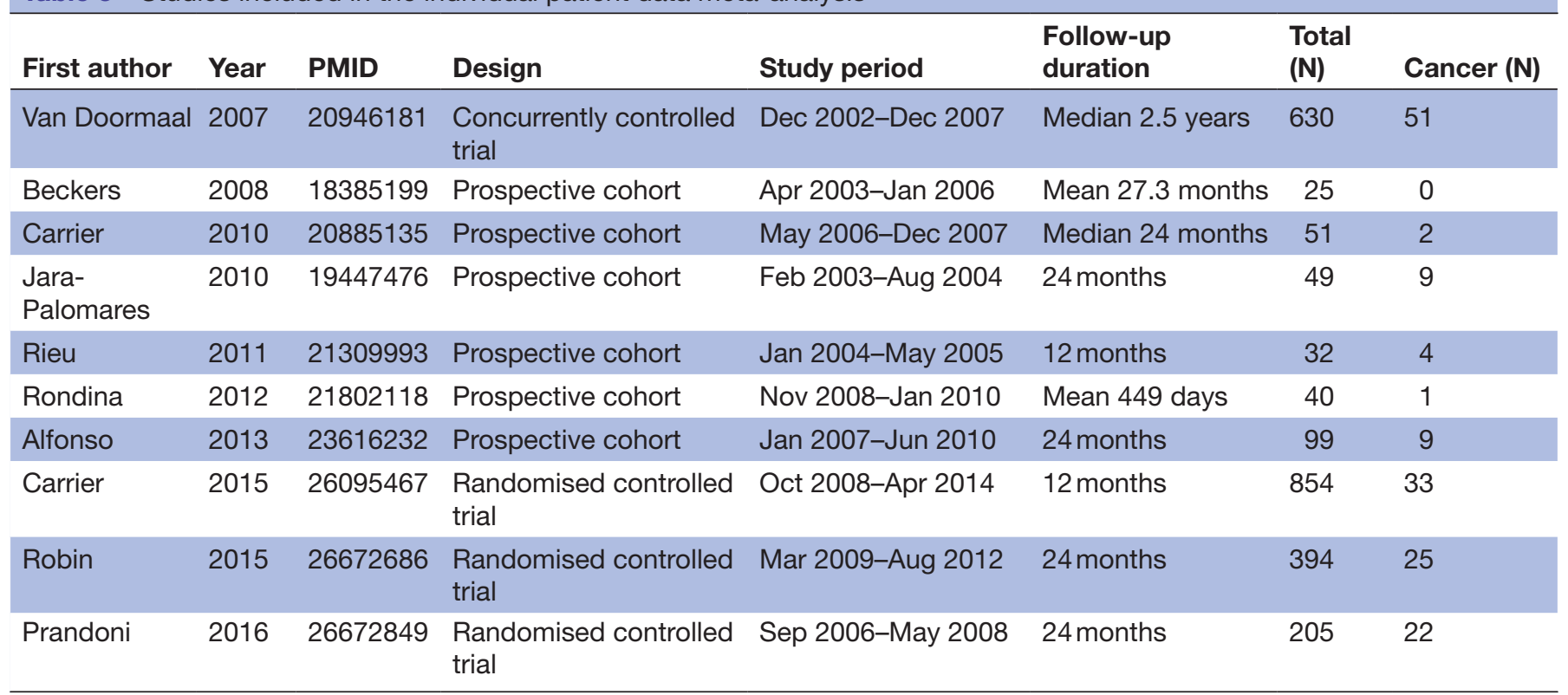


Reasons for exclusion of articles after full-text review are provided in table 2. Search results are summarised in figure 1. In accordance with the guidelines, this systematic review was registered with the International Prospective Register of Systematic Reviews on 19 January 2016 and was last updated on 29 September 2016 (registration number: CRD42016033371).

\section{Included studies}

The search identified 10 eligible studies ${ }^{1-49-14}$ (table 3). Corresponding authors were invited by email to participate in this project. Investigators of all 10 studies agreed to participation and were requested to provide their dataset. Patients who were enrolled more than 90 days after the index VTE were excluded from the dataset as well as those in whom the index VTE was not confirmed. Investigators were provided with a template file of the common dataset including variable definitions. In all studies, patients provided oral or written informed consent.

\section{Outcomes}

The following outcome definitions were used.

Primary outcome: haematological or solid cancer (other than basal cell or squamous cell carcinoma of the skin), objectively confirmed by histology or cytology, or unequivocally diagnosed by imaging or tumour markers.

Secondary outcomes:

- Objectively confirmed early stage solid cancer (other than basal cell or squamous cell carcinoma of the skin), defined as stage I or II according to the American Joint Committee on Cancer staging system;

- Objectively confirmed solid cancer (other than basal cell or squamous cell carcinoma of the skin) with distant metastasis

- Positive result, false positive result and true positive result of the limited occult cancer screening strategy as a whole (medical history, physical examination, basic blood work, chest X-ray and/or age-specific and gender-specific testing) and of individual limited or extensive screening tests;

- All-cause mortality;

- Cancer-related mortality according to the study definitions;

- Recurrent VTE according to the study definitions.

\section{Risk of bias of individual studies}

The Quality Assessment of Diagnostic Accuracy Studies (QUADAS)-2 tool was used for the risk of bias assessment. Signalling questions for each domain was adapted or omitted, and we added questions, if needed. Two reviewers independently assessed the studies for risks of bias on a study level. A judgement as to the possible risk of bias on each item in the domains ('low risk', 'unclear' or 'high risk') was made from study-level data and, if needed, from a summary of the obtained individual patient data. Results were compared and disagreements resolved by discussion or, if needed, with the help of a third reviewer.

\section{Research questions}

Research question 1

What is the absolute risk of cancer in the first year after unprovoked VTE, in the second year after unprovoked VTE and after an initial negative limited screening?

The primary objective was to estimate the absolute 1-year risk of occult cancer detection, defined as the number of patients with newly diagnosed cancer during a 12-month follow-up period in all patients with unprovoked VTE. In addition, the risk of occult cancer in the second year was estimated, defined as the number of patients with newly diagnosed cancer between 12 and 24 months of follow-up, in patients who were not diagnosed with cancer, did not die and were not lost to follow-up in the first year. We also estimated the risk of occult cancer after a negative occult cancer screening, defined as the number of patients with newly diagnosed cancer after a negative screening until 12 months follow-up.

\section{Research question 2}

What are the positive predictive value and false positive rate of a limited screening strategy and what is the discriminatory performance of the different limited screening components and diagnostic tests?

Limited screening was defined as the combination of medical history, physical examination, basic blood work (complete blood count and renal and liver function testing), a chest X-ray and/or age-specific and gender-specific testing. Using the information in the database and, if necessary, source data, patients allocated to limited screening were classified as having a negative or positive limited screening. A negative limited screening was defined as a screening that did not require additional targeted testing, while a positive limited screening was defined as a screening leading to additional testing. In addition, we evaluated which index screenings tests eventually led to the final cancer diagnosis.

Furthermore, the discriminatory performance of objective tests (eg, blood tests and chest X-ray) was evaluated by calculating the area under the ROC curve for continuous tests and diagnostic ORs based on $2 \times 2$ contingency tables for dichotomous tests.

\section{Research question 3}

Which patients are at high risk of occult cancer and can they be identified by a risk prediction model?

We planned multiple subgroup analyses; these were selected because they are clinically plausible and there is evidence that they might be relevant. ${ }^{15}$ We proposed the following subgroup analyses:

- Male versus female

- Age $<50$ vs age $\geq 50$

- Age $<75$ vs age $\geq 75$

- Age cohorts stratified by 10 years

- Previous cancer versus no previous cancer

- Previous VTE versus no previous VTE

- Index DVT versus index PE \pm DVT

- Oestrogen use versus no oestrogen use 
- Current/former smoker versus never smoker

- Patients receiving any form of extensive screening versus limited screening only

In addition, we validated the performance of existing risk prediction scores or models for cancer in patients with unprovoked VTE, ${ }^{15}$ as well as explored the performance of a new risk prediction model to identify high-risk patients. A multivariable generalised linear mixed-effects model with cancer diagnosis during 1-year follow-up period as the dependent variable was built. We considered the following candidate predictors, if sufficient overlap across the studies: age (continuous), gender (male vs female), body weight (continuous), previous cancer (yes vs no), previous VTE (yes vs no), type of VTE (PE \pm DVT vs single leg DVT vs bilateral DVT), D-dimer level (continuous), smoking status (ever smoked vs never smoked), current oestrogen use (yes vs no), haemoglobin (continuous), white blood cell count (continuous), platelet count (continuous), aspartate transaminase continuous), alanine transaminase continuous), gamma-glutamyl transferase (continuous), alkaline phosphatase (continuous), bilirubin (continuous), calcium (continuous) and lactate dehydrogenase (continuous). Restricted cubic splines with four knots were used to explore non-linear associations of continuous variables. Using manual stepwise backward selection, we arrived at the final parsimonious model. The model was internally validated by refitting the model in bootstrap samples. Coefficients were shrunk according to the amount of overoptimism in the coefficients and the intercept was subsequently adjusted. The performance of the model was evaluated by the c-statistic and Nagelkerke's $R^{2}$. Calibration was visually inspected by a calibration plot and evaluated using the Hosmer-Lemeshow goodness-of-fit test.

\section{Research question 4}

Is long-term all-cause mortality lower in patients undergoing an extensive occult cancer screening strategy compared with those undergoing a limited screening?

Data from three trials (ie, SOME, ${ }^{3}$ Trousseau ${ }^{2}$ and MVTEP $^{1}$ ) were used to address this question, since these studies compared limited and extensive screening in patients who had not undergone previous screening. Follow-up data of cancer patients were obtained through medical records and/or by telephone contact to ascertain the clinical status of the patients. The cumulative risk of mortality from the date of inclusion was estimated using the KaplanMeier estimator, censoring patients at the date of last follow-up contact or loss to follow-up. The difference between the two groups was assessed by a two-stage meta-analysis of the study-specific HRs obtained by a Cox proportional hazards regression model. The proportionality assumption was checked by visually inspecting the log-minus-log plot.
Research question 5

What types and stages of cancer are detected in patients with unprovoked VTE and by which index screening test(s) are they diagnosed?

The TNM classification was obtained for patients who were diagnosed with solid cancer and they were classified based on the American Joint Committee on Cancer staging system. Using this information, patients were further classified as metastatic versus no metastatic disease, early stage (I or II) versus late-stage (III or IV) cancer and curable versus potentially curable versus incurable cancer. Information about haematological cancers was only summarised. Based on the information in the databases and, if necessary, source data, the screening test or tests that led to the diagnosis of cancer were identified.

\section{Research question 6}

What is the diagnostic accuracy of the various diagnostic components of an extensive screening strategy (CT chest, CT abdomen, ultrasound abdomen and whole-body PET/CT)?

Among patients allocated to extensive screening, we calculated the (1) positive predictive value of various imaging tests, defined as the number of patients with cancer with a positive imaging test result, relative to all patients with a positive imaging test result, (2) negative predictive value, defined as the number of patients without cancer and negative imaging test result, relative to all patients with a negative test result, (3) sensitivity, defined as the number of patients with cancer with a positive imaging test result, relative to all patients in whom cancer is detected and (4) specificity, defined as the number of patients without cancer and a negative imaging test result, relative to all patients without cancer being detected.

\section{Data synthesis}

The dataset included five main data categories: demographics, index VTE, cancer screening tests, cancer diagnosis and follow-up information. Once the de-identified individual patient data from all primary studies were homogenised and merged, descriptive statistics were used to check consistency of the data. Using the provided datasets, the baseline tables and primary analysis were replicated. Any inconsistencies or discrepancies were resolved by contacting the investigators.

\section{Statistical analysis}

Data were quantitatively synthesised as follows. First, a two-stage meta-analysis was performed using the complete case database for all outcomes to generate forest plots, enabling results across studies to be compared visually, illustrate heterogeneity and differences across subgroups. $^{17}$ Next, a one-stage meta-analysis was performed using generalised linear mixed-effects models to generate the final estimates. This approach allowed for a more flexible environment in case of imputed datasets and was easier to use when evaluating multiple covariates, 


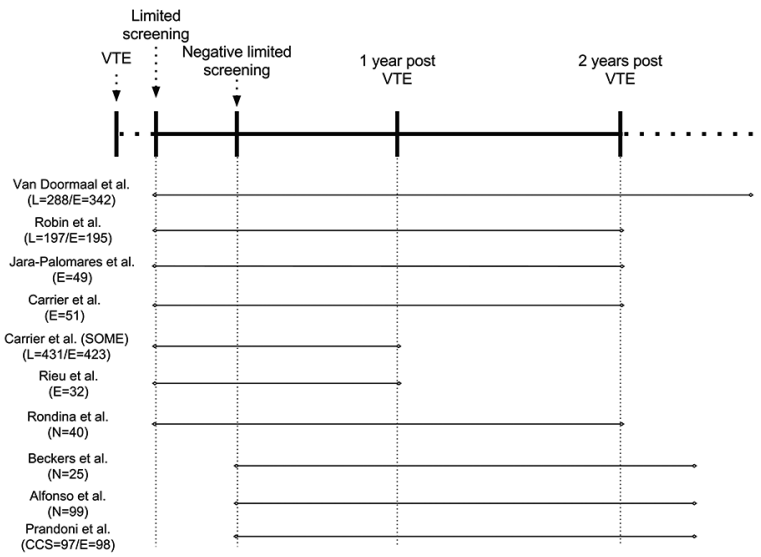

Figure 2 Overview of the design and duration of the eligible studies. L, limited screening; E, extensive screening; CCP, common clinical practice; VTE, venous thromboembolism.

covariate interactions, continuous variables or non-linear relationships. ${ }^{17}$ In addition, we felt that a one-stage meta-analysis was more appropriate for the present IPDMA than two-stage meta-analysis since the expected number of included studies and participants was small and clinical heterogeneity with respect to intervention strategies was expected. ${ }^{17}$ Different subsets of studies may be used to address the various research questions based on study characteristics, design or duration (figure 2).

The generalised linear mixed-effects models was built using the lme 4 package in statistical software $\mathrm{R}$, with the binomial outcome of interest as the dependent variable. Since observations within a single study are more similar than observations between different studies, a study-specific random effect for the intercept in the model was specified to account for this clustering. ${ }^{18}$ This implies that a different baseline risk for the outcome variable is assumed for every study. The model estimates both a random effect (variance of the intercept) and fixed effects (log-odds for the intercept and covariates). To estimate the mean population-averaged (or marginal) predicted probability, the inverse logit of the fixed effects was integrated over the random-effects distribution. ${ }^{19}$ Because this integral cannot be analytically solved, Gauss-Hermite quadrature approximation with 10 quadrature points was used to estimate the point estimates and $95 \%$ CIs using the spatstat package in $\mathrm{R}$.

\section{Dealing with missing data}

Multiple imputation was used to minimise the bias associated with missing data. ${ }^{20}$ Differences between patients with and without missing data were explored. Under the assumption of a missing at random pattern, multiple imputation was performed 20 times within each study database using the mice package in R. Only variables that had $25 \%$ or less missing values were subjected to imputation. Variables considered to guide the multiple imputation process included (but were not limited to) age, sex, type of VTE, previous VTE, oestrogen use, smoking status, mild provoking factors, results of screening tests, cancer diagnosis, cancer stage, recurrent VTE and death. After within-study multiple imputations, the imputed datasets were merged into a single individual patient database. The results of the models across the multiply imputed datasets were combined using Rubin's rule.

\section{Assessment of heterogeneity}

To illustrate the heterogeneity between the studies, we calculated $95 \%$ prediction intervals around the point estimates in all analyses. These intervals indicate the range of possible estimates that can be expected in a future study. The $95 \%$ upper and lower bounds of the prediction interval were calculated by multiplying the 0.025 or 0.975 percentile of the Student's T distribution (with df of the number of studies minus 2), with the square root of the sum of the variances of the random effects and fixed effects. ${ }^{18}$ We have tried to explain the source of heterogeneity by subgroup or sensitivity analysis.

\section{Sensitivity analyses}

The following sensitivity analyses were conducted to evaluate the robustness of the results: complete case analysis versus multiple imputation analysis; patients enrolled within 30 days after the index VTE versus those enrolled between 30 and 90 days after the index VTE, and low risk of bias versus unclear/high risk of bias according to QUADAS-2 results. Sensitivity analyses based on study period, referral setting, number of centres and geographic region were considered.

\section{Limitations and challenges}

IPDMA is a powerful method to address questions, since combining individual data from multiple studies allows for greater precision of estimates, analysis of clinically relevant subgroups and the evaluation of narrower outcomes (eg, early stage cancer). In addition, an IPDMA enables exploration of methodological and statistical heterogeneity between the studies. However, IPDMA also have limitations that need to be highlighted.

Pooling of data may be biassed due to differences across the studies with respect to inclusion criteria, time from VTE to enrolment and definitions of baseline and outcome variables. For example, patients with minor provoking factors (eg, oestrogen therapy and long-haul travel) were included in some studies, while others excluded those patients. Although all investigators were provided with a template of the common dataset including variable definitions, we acknowledge that it will often not be possible to retrieve additional information from the medical records. Similarly, we are well aware that it may be difficult, and for some studies impossible, to retrieve accurate information about test results, cancer diagnosis and index tests that led to cancer detection. As a consequence, analyses may be restricted to subgroups of studies which can provide the required information.

The potential benefit of extensive screening over limited screening has been heavily debated over the past two decades. Now that all contemporary studies that addressed this topic will be analysed in this IPDMA, it is tempting to 
compare the performance of the extensive and limited screening strategies. However, such a pooled analysis is hampered by clinical heterogeneity, since extensive screening strategies may vary considerably between studies.

Several studies have excluded patients with a positive limited screening at baseline. Information about the limited screening test results in these patients may be lacking. We anticipate that these data, when available, may potentially be subject to selection bias, as patients with cancer detected by limited screening are more likely to be reported by physicians, and testing may not have been done per protocol. We therefore aim to restrict the analysis of limited screening tests to those studies which enrolled unselected patients with unprovoked VTE.

\section{ETHICS AND DISSEMINATION}

By bringing together individual participant data from 10 contemporary studies that have evaluated screening for cancer in patients with unprovoked VTE, the present IPDMA aims to address several unanswered questions about the absolute risk of occult cancer, the performance and potential benefit of screening tests and identification of high-risk patients. Ethical approval was not required since similar research questions as in the original studies were addressed and no primary data were collected. Findings from this study have been submitted for publication in peer-reviewed journals and presentation at national and international conferences to inform clinicians and decision-makers.

\section{Author affiliations}

${ }^{1}$ Department of Vascular Medicine, Academic Medical Center, Amsterdam, The Netherlands

${ }^{2}$ Department of Medicine, Ottawa Hospital Research Institute, Ottawa, Canada ${ }^{3}$ Département de Médecine Interne et Pneumologie, Centre Hospitalo-Universitaire de Brest, Université de Bretagne Occidentale, Brest, France

${ }^{4}$ Department of Internal Medicine, Slotervaartziekenhuis, Amsterdam, The Netherlands

${ }^{5}$ Service de Médecine Nucléaire, Centre Hospitalo-Universitaire de Brest, Université de Bretagne Occidentale, Brest, France

${ }^{6}$ Departments of Cardiovascular Sciences and Medicine, University Hospital of Padua, Padua, Italy

${ }^{7}$ Hematology Service, Clinica Universidad de Navarra, Pamplona, Spain

${ }^{8}$ Medical Surgical Unit of Respiratory Diseases. Instituto de Biomedicina de Sevilla (IBiS), Centro de Investigación Biomédica en Red de Enfermedades Respiratorias (CIBERES), Sevilla, Spain

${ }^{9}$ Department of Medicine, Karolinska Institutet, Stockholm, Sweden

${ }^{10}$ Department of Medicine, Warsaw Medical University, Warsaw, Poland

${ }^{11}$ Department of Internal Medicine, CHU Estaing, Clermont-Ferrand, France

${ }^{12}$ Division of General Internal Medicine, University of Utah, Salt Lake City, Utah, USA

${ }^{13}$ Department of Hematology, University Hospital Leuven, Leuven, Belgium

${ }^{14}$ Dipartimento di Medicina e Scienze dell Invecchiamento, Università 'Gabriele

d'Annunzio', Chieti-Pescara, Italy

${ }^{15}$ Department of Clinical Epidemiology, Biostatistics, and Bioinformatics, Academic Medical Center, Amsterdam, The Netherlands

Contributors NvE is the guarantor. NvE, GLG and MC drafted the manuscript. $\mathrm{NvE}, \mathrm{MDN}, \mathrm{H}-\mathrm{MO}, \mathrm{MC}$ and $\mathrm{HRB}$ developed the selection criteria. NvE, MC and PMB developed the risk of bias strategy. NvE developed the search strategy. All authors read, provided feedback and approved the final manuscript.

Competing interests None declared.

Provenance and peer review Not commissioned; externally peer reviewed.
Open Access This is an Open Access article distributed in accordance with the Creative Commons Attribution Non Commercial (CC BY-NC 4.0) license, which permits others to distribute, remix, adapt, build upon this work non-commercially, and license their derivative works on different terms, provided the original work is properly cited and the use is non-commercial. See: http://creativecommons.org/ licenses/by-nc/4.0/

(C) Article author(s) (or their employer(s) unless otherwise stated in the text of the article) 2017. All rights reserved. No commercial use is permitted unless otherwise expressly granted.

\section{REFERENCES}

1. Robin P, Le Roux PY, Planquette B, et al. Limited screening with versus without (18)F-fluorodeoxyglucose PET/CT for occult malignancy in unprovoked venous thromboembolism: an open-label randomised controlled trial. Lancet Oncol 2016;17:1-7.

2. Van Doormaal FF, Terpstra W, Van Der Griend R, et al. Is extensive screening for Cancer in idiopathic venous thromboembolism warranted? J Thromb Haemost 2011;9:79-84.

3. Carrier M, Lazo-Langner A, Shivakumar S, et al. Screening for Occult Cancer in Unprovoked venous thromboembolism. N Engl J Med Overseas Ed 2015;373:697-704.

4. Prandoni P, Bernardi E, Valle FD, et al. Extensive Computed Tomography versus Limited Screening for detection of Occult Cancer in Unprovoked venous thromboembolism: a Multicenter, Controlled, Randomized clinical trial. Semin Thromb Hemost 2016;42.

5. Khorana AA, Carrier M, Garcia DA, et al. Guidance for the prevention and treatment of cancer-associated venous thromboembolism. J Thromb Thrombolysis 2016;41:81-91.

6. Moher D, Shamseer L, Clarke M, et al. Preferred reporting items for systematic review and meta-analysis protocols (PRISMA-P) 2015 statement. Syst Rev 2015;4:1.

7. Stewart LA, Clarke M, Rovers M, et al. Preferred Reporting items for systematic review and Meta-Analyses of individual participant data: the PRISMA-IPD statement. JAMA 2015;313:1657.

8. Carrier M, Le Gal G, Wells PS, et al. Systematic review: the trousseau syndrome revisited: should we screen extensively for Cancer in patients with venous thromboembolism? Ann Intern Med 2008;149:323-33.

9. Carrier M, Le Gal G, Tao H, et al. Should we screen patients with unprovoked venous thromboembolism for occult cancers? A pilot study. Blood Coagul Fibrinolysis 2010;21:709-10.

10. Alfonso A, Redondo M, Rubio T, et al. Screening for occult malignancy with FDG-PET/CT in patients with unprovoked venous thromboembolism. Int J Cancer 2013;133:2157-64.

11. Rondina MT, Wanner N, Pendleton RC, et al. A pilot study utilizing whole body 18 F-FDG-PET/CT as a comprehensive screening strategy for occult malignancy in patients with unprovoked venous thromboembolism. Thromb Res 2012;129:22-7.

12. Beckers MM, Verzijlbergen JF, van Buul MM, et al. The potential role of positron emission tomography in the detection of occult Cancer in 25 patients with venous thromboembolism. Ann Oncol 2008;19:1203-4.

13. Jara-Palomares L, Rodríguez-Matute C, Elías-Hernández T, et al. Testing for occult Cancer in patients with pulmonary embolism: results from a screening program and a two-year follow-up survey. Thromb Res 2010;125:29-33.

14. Rieu V, Chanier S, Philippe P, et al. Systematic screening for occult Cancer in elderly patients with venous thromboembolism: a prospective study. Intern Med J 2011;41:769-75.

15. Ihaddadene R, Corsi DJ, Lazo-Langner A, et al. Risk factors predictive of occult Cancer detection in patients with unprovoked venous thromboembolism. Blood 2016:127:2035-7.

16. Jara-Palomares L, Otero R, Jimenez D, et al. Development of a risk prediction score for Occult Cancer in Patients with VTE. Chest 2017; 151.

17. Stewart GB, Altman DG, Askie LM, et al. Statistical analysis of individual participant data meta-analyses: a comparison of methods and recommendations for practice. PLoS One 2012;7:e46042.

18. Debray TP, Moons KG, Abo-Zaid GM, et al. Individual participant data meta-analysis for a binary outcome: one-stage or two-stage? PLoS One 2013;8:e60650.

19. Skrondal A, Rabe-Hesketh S. Prediction in multilevel generalized linear models. J R Stat Soc Ser A Stat Soc 2009;172:659-87.

20. van der Heijden GJ, Donders AR, Stijnen T, et al. Imputation of missing values is superior to complete case analysis and the missing-indicator method in multivariable diagnostic research: a clinical example. J Clin Epidemiol 2006;59:1102-9. 


\section{Correction: Screening for cancer in patients with}

unprovoked venous thromboembolism: protocol for a systematic review and individual patient data meta-analysis

van Es N, Gal GL, Otten H, et al. Screening for cancer in patients with unprovoked venous thromboembolism: protocol for a systematic review and individual patient data meta-analysis. BMJ Open 2017;7:e015562. doi: 10.1136/bmjopen-2016-015562

The author name 'Luis Jara Palomares' should be spelled 'Luis Jara-Palomares' where 'Luis' is the first name and 'Jara-Palomares' is the surname.

Open Access This is an Open Access article distributed in accordance with the Creative Commons Attribution Non Commercial (CC BY-NC 4.0) license, which permits others to distribute, remix, adapt, build upon this work non-commercially, and license their derivative works on different terms, provided the original work is properly cited and the use is non-commercial. See: http://creativecommons.org/licenses/by-nc/4.0/

(C) Article author(s) (or their employer(s) unless otherwise stated in the text of the article) 2017. All rights reserved. No commercial use is permitted unless otherwise expressly granted.

BMJ Open 2017;7:e015562corr1. doi:10.1136/bmjopen-2016-015562corr1

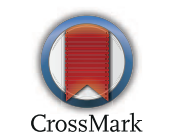

